\title{
Identification of a quantitative trait loci (QTL) associated with ammonia tolerance in the Pacific white shrimp (Litopenaeus vannamei)
}

Digang Zeng ${ }^{1 \dagger}$, Chunling Yang ${ }^{1 \dagger}$, Qiangyong Li ${ }^{1}$, Weilin Zhu ${ }^{1}$, Xiuli Chen ${ }^{1}$, Min Peng ${ }^{1}$, Xiaohan Chen ${ }^{1}$, Yong Lin ${ }^{1}$, Huanling Wang ${ }^{2}$, Hong Liü ${ }^{2}$, Jingzhen Liang ${ }^{3}$, Qingyun Liü ${ }^{1^{*}}$ and Yongzhen Zhao ${ }^{*^{*}}$ (D)

\begin{abstract}
Background: Ammonia is one of the most common toxicological environment factors affecting shrimp health. Although ammonia tolerance in shrimp is closely related to successful industrial production, few genetic studies of this trait are available.

Results: In this study, we constructed a high-density genetic map of the Pacific white shrimp (Litopenaeus vannamei) using specific length amplified fragment sequencing (SLAF-seq). The constructed genetic map contained 17,338 polymorphic markers spanning 44 linkage groups, with a total distance of 6360.12 centimorgans (cM) and an average distance of $0.37 \mathrm{cM}$. Using this genetic map, we identified a quantitative trait locus (QTL) that explained $7.41-8.46 \%$ of the phenotypic variance in L. vannamei survival time under acute ammonia stress. We then sequenced the transcriptomes of the most ammonia-tolerant and the most ammonia-sensitive individuals from each of four genetically distinct $L$. vannamei families. We found that 7546 genes were differentially expressed between the ammonia-tolerant and ammonia-sensitive individuals. Using QTL analysis and the transcriptomes, we identified one candidate gene (annotated as an ATP synthase $g$ subunit) associated with ammonia tolerance.

Conclusions: In this study, we constructed a high-density genetic map of L. vannamei and identified a QTL for ammonia tolerance. By combining QTL and transcriptome analyses, we identified a candidate gene associated with ammonia tolerance. Our work provides the basis for future genetic studies focused on molecular marker-assisted selective breeding.
\end{abstract}

Keywords: Genetic map, QTL, Transcriptomic, Ammonia tolerance, Litopenaeus vannamei

\section{Background}

The Pacific white shrimp (Litopenaeus vannamei) is the most widely cultivated and highest-yielding crustacean species in the world [1]. L. vannamei tolerates a wide range of salinities, grows rapidly, is highly disease resistant, and can be farmed at high densities [2]. However,

\footnotetext{
*Correspondence: 1624935761@qq.com; yongzhenzhao@hotmail.com †Digang Zeng and Chunling Yang contributed equally to this work. ${ }^{1}$ Guangxi Key Laboratory of Aquatic Genetic Breeding and Healthy Aquaculture, Guangxi Academy of Fishery Sciences, Nanning 530021, China Full list of author information is available at the end of the article
}

high-density shrimp cultivation often leads to water quality deterioration [3]. The toxicological factors associated with poor quality water often negatively affect shrimp [4]. One of the most common toxicological factors affecting shrimp health is ammonia nitrogen (ammonia- $\mathrm{N}$ ) [5]. In aquaculture water, ammonia- $\mathrm{N}$ is mainly found as non-ionic ammonia $\left(\mathrm{NH}_{3}\right)$ and ionic ammonia $\left(\mathrm{NH}_{4}{ }^{+}\right)$; these compounds are usually in dynamic equilibrium [6]. As $\mathrm{NH}_{3}$ has no electric charge, it is highly fat-soluble and can easily penetrate organismal

(c) The Author(s). 2020 Open Access This article is licensed under a Creative Commons Attribution 4.0 International License, which permits use, sharing, adaptation, distribution and reproduction in any medium or format, as long as you give appropriate credit to the original author(s) and the source, provide a link to the Creative Commons licence, and indicate if changes were made. The images or other third party material in this article are included in the article's Creative Commons licence, unless indicated otherwise in a credit line to the material. If material is not included in the article's Creative Commons licence and your intended use is not permitted by statutory regulation or exceeds the permitted use, you will need to obtain permission directly from the copyright holder. To view a copy of this licence, visit http://creativecommons.org/licenses/by/4.0/ The Creative Commons Public Domain Dedication waiver (http://creativecommons.org/publicdomain/zero/1.0/) applies to the data made available in this article, unless otherwise stated in a credit line to the data. 
cell membranes, leading to toxic effects [7]. In aquatic organisms, $\mathrm{NH}_{3}$ affects membrane stability, as well as physiology, biochemistry, and growth; shrimp exposed to $\mathrm{NH}_{3}$ may exhibit dyspnea, lack of appetite, decreased disease resistance, and even death [8-11].

The maintenance of low aquatic ammonia- $\mathrm{N}$ concentrations is required for successful shrimp farming [12]. However, aquatic physical and chemical properties are complicated, and may be affected by various factors such as weather, the local environment, and the introduction of artificial feeds. Thus, new breeds of ammonia-tolerant shrimp may improve industrial production and reduce economic losses. Marker-assisted selection has proven to be a useful strategy for the development of new breeds with dramatically improved trait characteristics [13], and the first step towards developing a new shrimp breed is to identify genes or markers associated with the desired trait [14]. Several previous studies have focused on the genetic bases of ammonia tolerance in shrimp. For example, Lu et al. identified 12 single nucleotide polymorphisms (SNPs) associated with ammonia tolerance in $L$. vannamei using marker-trait correlation analyses [15]. At the same time, $\mathrm{Lu}$ et al. identified 202 proteins that were significantly differentially expressed between ammonia-tolerant and ammonia-sensitive L. vannamei families using a comparative proteome analysis based on isobaric tags for relative and absolute quantification (iTRAQ) [16]. In addition, Jie et al. identified several pathways and genes involved in ammonia tolerance in $L$. vannamei based on comparative transcriptomic and metabolomic analyses of ammonia-tolerant and ammonia-sensitive L. vannamei families [17]. Finally, several studies identified transcriptomic changes and differentially expressed genes in L. vannamei after ammonia stress $[9,18]$. However, no studies have investigated the quantitative trait loci (QTL) associated with ammonia tolerance in shrimp.

QTL analysis effectively identifies molecular markers or candidate genes associated with economically important traits in plants and animals [19]. QTL analyses usually require high-density genetic linkage maps. To date, genetic linkage maps have primarily been constructed using high-throughput sequencing technologies, such as restriction site-related DNA sequencing (RAD-seq), genotyping sequencing (GBS), and specific length amplified fragment sequencing (SLAF-seq) [20]. In particular, SLAF-seq efficiently identifies and genotypes large-scale SNPs [20]. SLAF-seq has been applied to many plant species, including spinach [21], sesame [22], walnut [23] soybean [24], cucumber [25], wax gourd [26], cauliflower [27], white jute [28], and maize [29]. SLAF-seq has also been successfully applied to L. vannamei [30].

Therefore, SLAF-seq was used in the current study to construct a high-density genetic map of $L$. vannamei.
Furthermore, QTL analysis of ammonia tolerance in $L$. vannamei was performed. Transcriptomic differences between ammonia-tolerant and ammonia-sensitive individuals across several $L$. vannamei families were compared to identify potential candidate genes coferring ammonia tolerance within QTLs.

\section{Methods \\ Preparation of the mapping family}

The $L$. vannamei used in experiments were obtained from the shrimp-breeding center at the Guangxi Academy of Fishery Sciences (Nanning, Guangxi, China). The L. vannamei family used for mapping was constructed using artificial insemination. In brief, a male shrimp from a family with a relatively high ammonia-tolerance (obtained via 10 consecutive generations of breeding) was mated with a female shrimp from a common family. The hatched offspring were reared for about 1 year. Then, a male and female shrimp were randomly selected from the year-old offspring and mated. The F1 progeny were used for mapping (LV-N).

\section{Measurement of ammonia tolerance}

A total of 284 shrimp (average body weight: $20.78 \mathrm{~g}$ ) were randomly selected from the LV-N family. Selected shrimp were transferred to a $2 \mathrm{~m} \times 4 \mathrm{~m} \times 1 \mathrm{~m}$ indoor pool and allowed to acclimate for 1 week. Aquatic conditions during the acclimation and experimental periods were kept constant: temperature of $27.0 \pm 0.5^{\circ} \mathrm{C}$, pH of $8.1 \pm 0.2$, salinity of $30.2 \%$, and dissolved oxygen of $6-8$ $\mathrm{mg} / \mathrm{L}$; culture water was kept aerated, and shrimp were fed formulated pellets (Zhengda Feed, China) daily at a ratio of $5 \%$ body weight. Following acclimation, an acute ammonia stress test was performed. The ammonia-N concentration used for the acute stress test was 345.94 $\mathrm{mg} / \mathrm{L}$, based on the results of a preliminary experiment. This was the concentration at which half of the experimental shrimp died in $72 \mathrm{~h}$ under stress. The ammonia$\mathrm{N}$ concentration of the water in the experimental pool was controlled by adding $\mathrm{NH}_{4} \mathrm{Cl}$ stock solution (prepared by dissolving analytically pure $\mathrm{NH}_{4} \mathrm{Cl}$ in filtered seawater). The concentration of ammonia- $\mathrm{N}$ in the water was measured daily using standard methods [31]. To keep the ammonia- $\mathrm{N}$ concentration constant, $\mathrm{NH}_{4} \mathrm{Cl}$ stock solution was added if the ammonia- $\mathrm{N}$ concentration was $<345.94 \mathrm{mg} / \mathrm{L}$, and seawater was added if the ammonia-N concentration was $>345.94 \mathrm{mg} / \mathrm{L}$. During the experiment, shrimp heath was observed every hour, and dead shrimp were removed immediately. Shrimp were considered dead when lying motionless on the bottom of the pool and not responding to external stimuli. Collected dead shrimp were immediately frozen in liquid nitrogen and stored at $-20^{\circ} \mathrm{C}$ for DNA extraction. The survival time of each shrimp was used as a proxy for 
ammonia tolerance. The experiment ended when all shrimp had died.

\section{DNA extraction}

DNA was collected from the 284 F1 (LV-N) shrimp and the two parent shrimp. Marine animal genomic DNA extraction kits (Tiangen Biotech, China) were used to extract DNA from the tail muscle of each shrimp. DNA was quantified using a NanoDrop spectrophotometer and $1 \%$ agarose gel electrophoresis with a lambda DNA standard.

\section{SLAF library preparation and sequencing}

First, we predicted the digestion of the $L$. vannamei genome (https://www.ncbi.nlm.nih.gov/genome/?term=Vannamei) [32] using self-developed software. We digested the extracted genomic DNA of all LV-N shrimp using the endonucleases identified by the predictive software. Then, dual-index sequencing adaptors were ligated to the DNA fragments obtained by digestion with T4 ligase, and the fragments were amplified using polymerase chain reactions (PCRs). PCR products (314-414 bp including the adaptor sequences) were purified and reamplified using PCR. SLAF sequencing was carried out on an Illumina HiSeq system, following the Illuminarecommended procedure. To assess the accuracy of library construction, the same library-construction and sequencing steps using the genome of Oryza sativa japonica as a control was performed. Library construction and sequencing were performed by Biomarker Technologies Corporation (Beijing, China).

\section{SLAF-seq data analysis and genotyping}

The raw sequencing reads were quality controlled by removing reads with a quality score $<20$. The remaining raw reads were grouped by individual based on the dualindex adaptor sequences. The dual-index adaptor and 5bp end sequences were then trimmed to obtain clean reads. The clean reads were mapped to the $L$. vannamei genome (https://www.ncbi.nlm.nih.gov/genome/?term= Vannamei) [32] using BWA [33]. Reads mapped to the same position with $>95 \%$ identity were considered the same SLAF. SNP-based polymorphic SLAF markers were identified by aligning reads from the same SLAF sequence. These polymorphic SLAF markers were then filtered by removing those with a parental sequencing depth less than 10-fold; those where the number of SNPs was $>5$; those where the proportion of genotypes covering offspring was $<70 \%$; and those with significant segregation distortion (chi-square test $P<0.05$ ). The remaining polymorphic SLAFs were classified into eight separate patterns: $a \mathrm{a} \times \mathrm{bb}, \mathrm{ab} \times \mathrm{cd}, \mathrm{cc} \times \mathrm{ab}, \mathrm{ab} \times \mathrm{cc}$, ef $\times$ eg, hk $\times$ hk, nn $\times$ np, and $\operatorname{lm} \times$ ll. Because the mapping population used in this study was an F1 population, the polymorphic SLAF with the pattern aa $\times$ bb was removed, and the remaining polymorphic SLAFs were used for the construction of the genetic map.

\section{Genetic map construction and QTL analysis}

After coding the genotypes of the polymorphic SLAF markers, the genetic map was constructed using the single-chain clustering algorithm in HighMap [34], with the probability $\log$ threshold set to $\geq 5.0$ and a maximum recombination rate of 0.4. The Kosanbi mapping function was used to convert percent recombination to genetic distance (cM). QTL analysis was conducted using the R/qtl software package [35]. The logarithm of odds (LOD) threshold was determined based on 1000 permutations $(P<0.05)$. The phenotypic variance explained by the QTL was estimated using the formula $1-10^{-2 \mathrm{LOD} / n}$, where $\mathrm{n}$ was the sample size [36].

Transcriptome sequencing, candidate gene identification and quantitative real-time PCR (qRT-PCR) verification

To identify differentially expressed genes (DEGs) between ammonia-tolerant and ammonia-sensitive $L$. vannamei, the transcriptomes of $4 \mathrm{~L}$. vannamei families were sequenced: the mapping family $(\mathrm{LV}-\mathrm{N})$ and three other randomly chosen common families (LV-A, LV-C, and LV-F) with different genetic backgrounds. Our previous analysis indicated that the 24-h median lethal concentration of $\mathrm{NH} 4 \mathrm{Cl}$ was $140.96 \mathrm{mg} / \mathrm{L}, 189.19 \mathrm{mg} / \mathrm{L}$, $117.88 \mathrm{mg} / \mathrm{L}$, and $137.26 \mathrm{mg} / \mathrm{L}$ for families LV-A, LV-C, LV-F and LV-N, respectively (Supplementary Material, Table S1). Two hundred shrimp from each family were randomly selected, and subjected to the acute ammonia stress test $(345.94 \mathrm{mg} / \mathrm{L}$ ammonia- $\mathrm{N})$, as described above. In each family, 20 shrimp with the longest survival times (i.e., the most ammonia tolerant) were collected, as were the 20 shrimp with the shortest survival times (i.e., the most ammonia sensitive). When collecting the ammonia-sensitive shrimp, specimens that were out of balance and lying on the bottom of the pool were judged to be dying, and were collected immediately, without waiting for death. The hepatopancreas of each shrimp was extracted, and hepatopancreases were pooled to form an ammonia-tolerant sample and an ammonia-sensitive sample per family.

Total RNA was extracted from each pooled sample using TRIzol reagent (Invitrogen, USA), following the manufacturer's instructions. Residual genomic DNA was removed with DNase I. RNA purity (OD260 / 280), concentration, and absorption peak were measured using a NanoDrop 2000. RNA integrity was assessed using an RNA Nano 6000 Assay Kit with an Agilent Bioanalyzer 2100. The isolated mRNA was divided into $100-400 \mathrm{bp}$ fragments using an RNA fragment reagent (Illumina, USA). cDNA libraries were then constructed using 
NEBNext Ultra RNA Library Prep Kits (Illumina, USA), following manufacturer's recommendations, and sequenced on an Illumina HiSeq system (Illumina, USA). Library construction and sequencing were performed by Biomarker Technologies Corporation (Beijing, China).

Raw sequencing reads were trimmed and filtered using in-house Perl scripts to remove adaptor sequences and low-quality reads; the Q20, Q30, GC-content, and sequence duplication levels of the clean data were calculated. Clean reads were then aligned to the L. vannamei genome (https://www.ncbi.nlm.nih.gov/genome/?term= Vannamei) [32] using Hisat2 2.1.0 (http://ccb.jhu.edu/ software/hisat2/index.shtml) [37]. Matched reads were counted to determine gene expression levels using the fragments per kilobase of transcript per million mapped reads (FPKM) method [38]. DEGs were identified using edger [39]. unigenes were considered differentially expressed when the false discovery rate (FDR) was $\leq 0.01$ and the fold change between groups was $>2$. DEGs were functionally annotated against the following databases: Non-Redundant protein sequences (NR) (ftp://ftp.ncbi. nih.gov/blast/db/), Protein family (Pfam) (http://pfam. xfam.org/), Clusters of Orthologous Groups (http:// www.ncbi.nlm.nih.gov/COG/), Swiss-Prot (http://www. uniprot.org/), Kyoto Encyclopedia of Genes and Genomes (KEGG) (http://www.genome.jp/kegg/), and Gene Ontology (GO) (http://www.geneontology.org/).

After obtaining DEGs, candidate genes among the DEGs were identified. We consider candidate genes associated with ammonia tolerance when (1) candidate genes located within the QTL interval; (2) candidate genes differentially expressed between the most ammonia-tolerant and the most ammonia-sensitive individuals in the mapping family ( $\mathrm{LV}-\mathrm{N})$; (3) the regulation pattern (up- or down-regulated expression) of candidate genes between the most ammonia-tolerant and the most ammonia-sensitive individuals was consistent across the four families of Litopenaeus vannamei (LV-A, LV-C, LV-F, and LV-N).

qRT-PCR was used to validate the RNA-seq results by quantifying the expression of the candidate gene (LOC113809108) in the ammonia-tolerant and ammonia-sensitive pooled samples from the $4 \mathrm{~L}$. vannamei families (LV-A, LV-C, LV-F, and LV-N). RNA-seq and qRT-PCRs analyses were carried out using the same samples. qRT-PCRs were performed using SYBR Premix Ex TaqTM II kits (TaKaRa, Japan), according to the manufacturer's instructions. The primer sets used to detect LOC113809108 gene expression levels were designed using the Primer Premier software (version 5.0) [40] as follows: 5'-ACTTGGGTGCTGTAGCTCAA-3' and 5'-CTCGACAGCAACCAGGGTAT-3'. L. vannamei 18S RNA was used as the internal reference gene; this gene was amplified using the primer sets as follows:
5'-GCCTGAGAAACGGCTACCACATC-3' and 5'GTAGTAGCGACGGGCGGTGTGT-3' [41]. The qRTPCR cycling program was as follows: preheating at $95^{\circ} \mathrm{C}$ for $30 \mathrm{~s}$, followed by 40 cycles of $95^{\circ} \mathrm{C}$ for $5 \mathrm{~s}$ and $60^{\circ} \mathrm{C}$ for $30 \mathrm{~s}$. The qRT-PCR was carried out at $95^{\circ} \mathrm{C}$ for $40 \mathrm{~s}$, $95^{\circ} \mathrm{C}$ for $5 \mathrm{~s}$, and $62^{\circ} \mathrm{C}$ for $30 \mathrm{~s}$ for 40 cycles. Three parallel qRT-PCRs were carried out for each sample. Relative gene expression levels were calculated using the 2$\Delta \Delta C$ T method [42].

\section{Results \\ Phenotypic variation}

We developed an ammonia-tolerant shrimp family (designated LV-N) for mapping, and subjected 284 LV-N shrimp to an acute ammonia stress test. All shrimp died within 2-98 h, with a mean survival time of $65 \mathrm{~h}$. Individual survival times were normally distributed and thus suitable for QTL detection. The accumulated mortality rate of shrimp is showed in Supplementary Material Fig. S1.

\section{SLAF-seq and genotyping}

Based on the digestive enzyme prediction using the reference genome of L. vannamei, HaeIII and Hpy166II were used to digest the genomic DNA of the $284 \mathrm{LV}-\mathrm{N}$ shrimp for SLAF library construction. SLAF sequencing generated 439.77 gigabases (Gb) of data, consisting of 2201 megabases $(\mathrm{Mb})$ of 100-bp paired-end reads. Across all reads, the average Q30 was $95.81 \%$, the average GC content was $40.60 \%$, and the GC distribution was normal (Table 1). The rice (Oryza sativa japonica) genome was used as a control to estimate the validity of the library construction. For the rice library, 343.21 Mb of data (1.72 Mb paired-end reads) were generated, with a Q30 of $95.81 \%$ and a GC content of $40.96 \%$. In L. vannamei, $57.83 \%$ of the paired-end reads mapped successfully to the genome, as compared to $91.43 \%$ of the paired-end reads in rice. In addition, enzymatic digestion efficiency was $87.75 \%$ for L. vannamei and $92.19 \%$ for rice (Supplementary Material, Table S2). These results indicated that SLAF library construction and sequencing were adequate.

After filtering and clustering all reads, 807,505 SLAFs were identified. The average sequencing depth of these SLAFs was 42.8-fold for the male parent, 42.14-fold for the female parent, and 12.43-fold for the progeny (Table 1). Of the 807,505 high-quality SLAFs detected, 293,415 (36.34\%) were polymorphic (Table 1). After further filtering, the remaining 115,973 SLAF markers were successfully classified into eight genotypic patterns: $a b \times c d$, $c c \times a b$, aa $\times$ bb, ab $\times$ cc, ef $\times$ eg, $\operatorname{lm} \times l l, h k \times h k$, and $\mathrm{nn} \times \mathrm{np}$. The most common pattern was aa $\times$ bb, followed by $\mathrm{nn} \times \mathrm{np}$ and $\operatorname{lm} \times \operatorname{ll}$ (Fig. 1). Because the mapped population was an F1 population, aa $\times$ bb were eliminated as a valid marker. 
Table 1 Summary of the constructed genetic map of Litopenaeus vannamei

\begin{tabular}{ll}
\hline Map data & Value \\
\hline Total bases & $439.77 \mathrm{~Gb}$ \\
Total reads & $2201.28 \mathrm{Mb}$ \\
Average Q30 & $95.81 \%$ \\
Average GC & $40.60 \%$ \\
Enzyme digestion protocol & Haelll+Hpy166II \\
Restriction fragment length & $314-414 \mathrm{bp}$ \\
Percentage of reads matching the L. vannamei & $57.83 \%$ \\
genome & \\
Average enzymatic digestion efficiency & $87.75 \%$ \\
Predicted number of markers & 339,517 \\
Number of high-quality slafs & 807,505 \\
Number of polymorphic slafs & 293,415 \\
Number of SLAF markers on the map & 17,338 \\
Average depth in parents & $208.90 \times$ \\
Average depth in offspring individual & $38.55 \times$ \\
Number of linkage groups & 44 \\
Total distance of the map & $6360.12 \mathrm{cM}$ \\
Average distance of the map & $0.37 \mathrm{cM}$ \\
\hline
\end{tabular}

\section{Characteristics of the genetic map}

Linkage analysis labeled 17,338 SLAF markers on the genetic map: 11,512 on the male map, 10,293 on the female map, and 17,338 on the sex-average map (Fig. 2). Each map contained 44 linkage groups (LGs). The total distances on the male, female, and sex-average maps were $6604.99 \mathrm{cM}, 5476.20 \mathrm{cM}$, and $6360.12 \mathrm{cM}$, respectively. The mean distance between adjacent markers was $0.58 \mathrm{cM}$ on the male map, $0.53 \mathrm{cM}$ on the female map, and $0.37 \mathrm{cM}$ on the sex-average map (Supplementary Material, Table S3, Table S4, and Table S5). The distribution of markers among LGs was not uniform: in the male map, LG31 contained the most markers (585), while LG26 contained the least (39); in the female map, LG36 contained the most markers (540), while LG27 contained the least (21); and in the sex-average map, LG31 contained the most markers (695), while LG26 contained the least (53).

\section{QTL mapping of ammonia-tolerance}

A QTL analysis of the ammonia-tolerance trait in the LV-N L. vannamei family was performed based on the genetic maps. The LOD threshold was 4.75 (1000 permutations, $P<0.05)$. Thus, QTLs with LOD scores $>$ 4.75 were considered effective QTLs. Using this criterion, we identified a QTL within LG19 for ammonia tolerance (Fig. 3). The phenotypic variation explained by this QTL was 7.41-8.46\%, the LOD score was 4.75-5.45, and the confidence interval was $12.42-29.43 \mathrm{cM}$.

\section{Transcriptome sequencing, candidate gene identification and $\mathrm{qRT}$-PCR verification}

The transcriptomes of the 20 most ammonia-tolerant and the 20 most ammonia-sensitive shrimp in each of $4 \mathrm{~L}$. vannamei families (LV-N, LV-A, LV-C, and LV-F) with various genetic backgrounds were sequenced. Transcriptome sequencing generated $56.79 \mathrm{~Gb}$ of clean data. A total of 7546 DEGs were identified between the ammoniatolerant and ammonia-sensitive shrimp across all four families: 1869 in LV-A, 2005 in LV-C, 1875 in LV-F, and 1797 in LV-N (Supplementary Material, Table S6).

The numbers of DEG annotations recovered in the databases searched were similar across the 4 L. vannamei

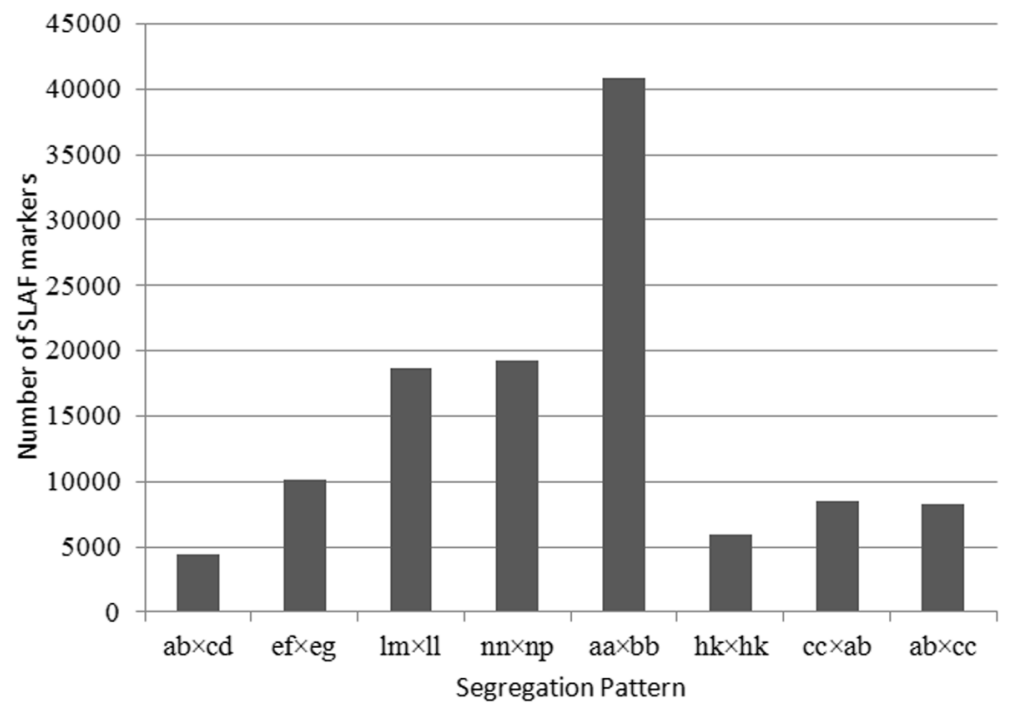

Fig. 1 Number of markers associated with each of the eight polymorphic specific length amplified fragment (SLAF) segregation patterns 


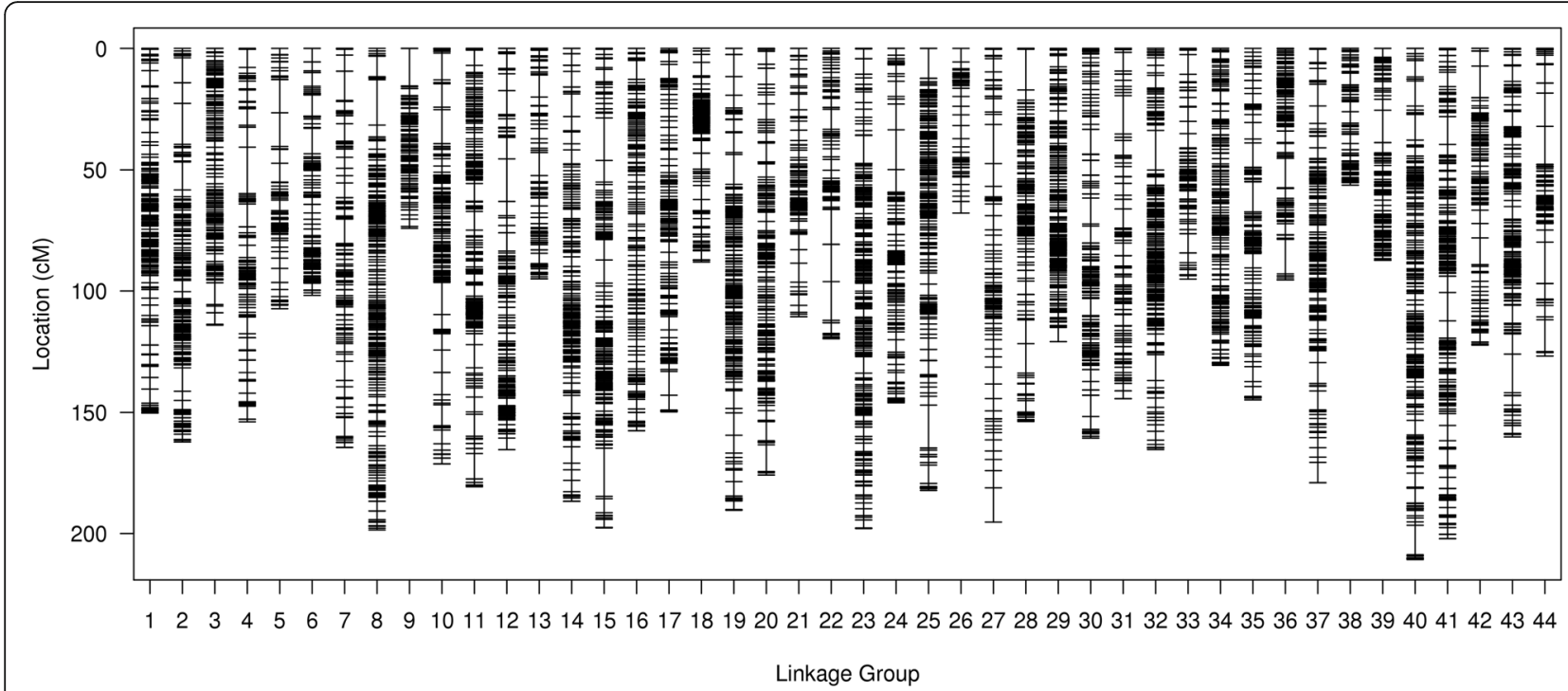

Fig. 2 High-density linkage map of Litopenaeus vannamei showing genetic distances among specific length amplified fragment (SLAF) markers. Black bars represent SLAF markers

families. For instance, the COG terms mainly enriched in the DEGs from all four families were posttranslational modification, protein turnover, chaperones, and general function prediction only (Fig. 4); the GO terms primarily enriched in the DEGs from all four families were binding, catalytic activity, cellular process, metabolic process, cell, cell part, single-organism process and membrane functions (Fig. 5).

By aligning the DEGs with the QTL region in LG19, 107 DEGs located in the QTL interval were identified. The expression levels and annotations of these DEGs are listed in
Supplementary Material Table S7. Of these DEGs, only one gene (LOC113809108) met the criterion used to determine candidate genes associated with ammonia tolerance. This gene was annotated as an ATP synthase $g$ subunit. LOC113809108 was located in the QTL interval, and was significantly upregulated in the most ammoniatolerant shrimp compared to the most ammonia-sensitive shrimp from families LV-N and LV-C (Fig. 6). This gene was also upregulated in the most ammonia-tolerant shrimp from families LV-A and LV-F, but this difference in expression was not significant (Table 2).

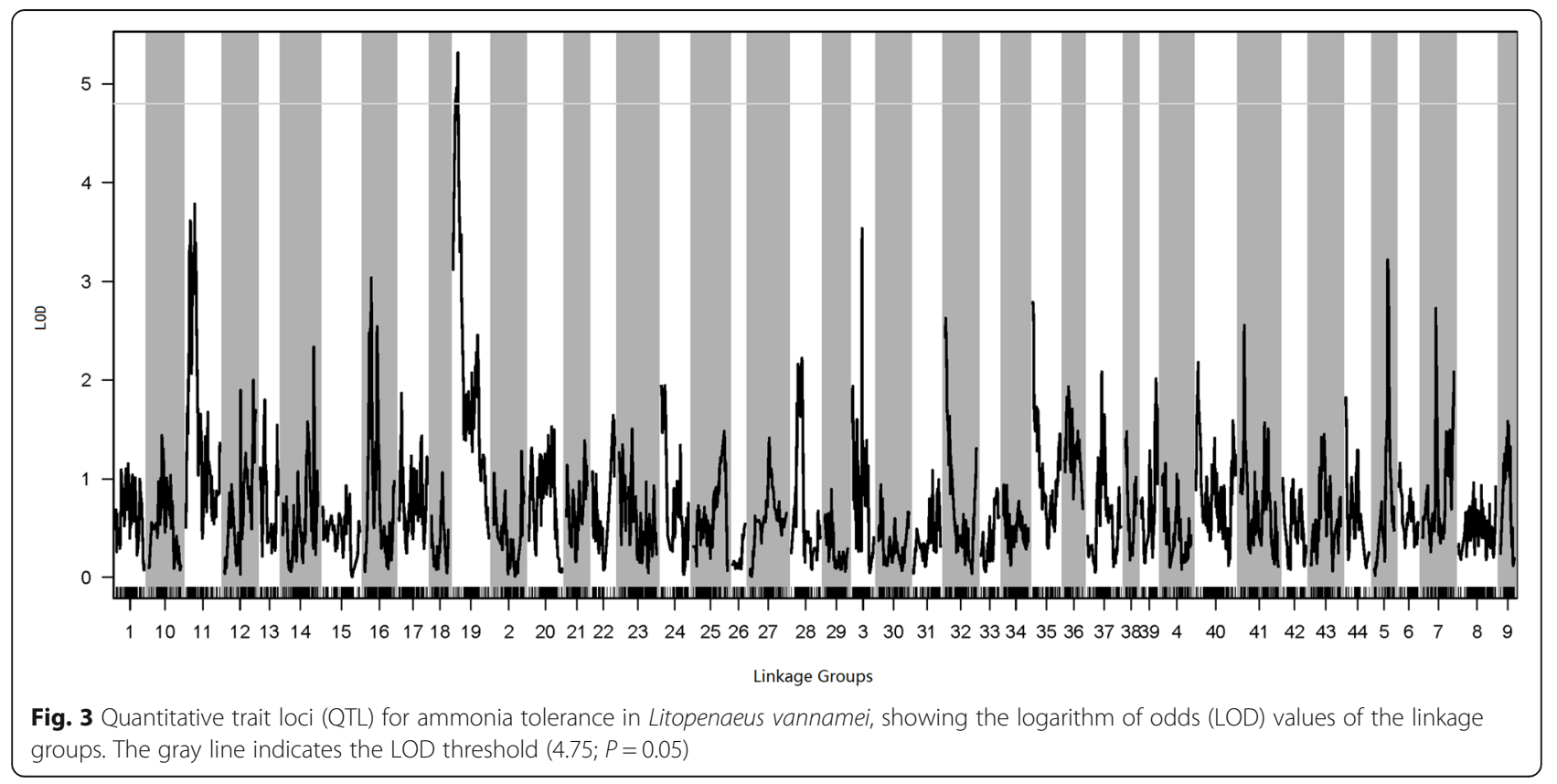




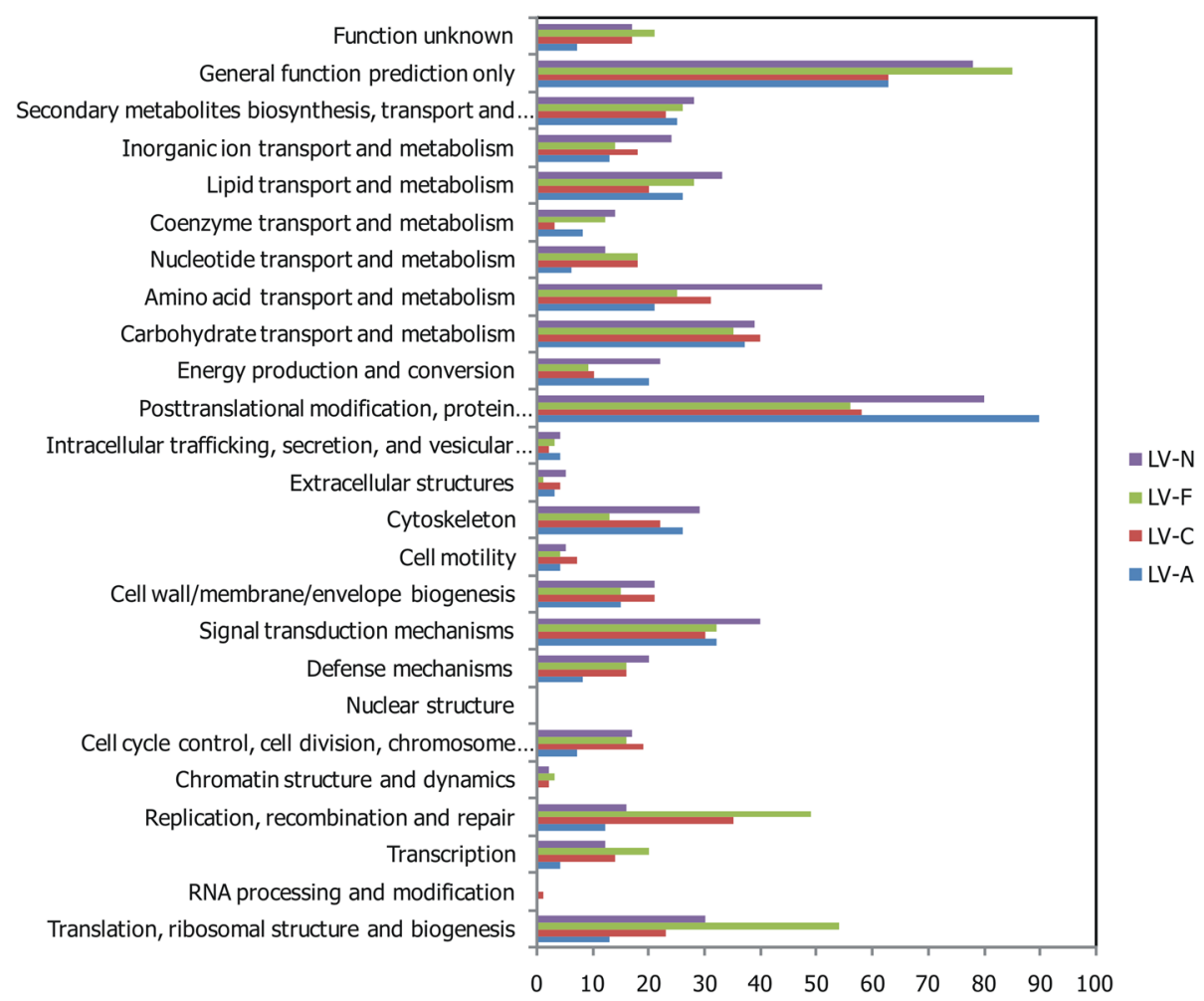

Fig. 4 Clusters of Orthologous Groups (COG) classifications of the putative functions of the differentially expressed genes between the most ammonia-tolerant and the most ammonia-sensitive individuals across four families of Litopenaeus vannamei (LV-A, LV-C, LV-F, and LV-N)

The qRT-PCR analysis showed that the patterns of LOC113809108 gene expression in ammonia-tolerant and ammonia-sensitive pooled samples from the families LV-A, LV-C, LV-F, and LV-N were similar to the patterns determined using RNA-seq: LOC113809108 gene expression was upregulated in the ammonia-tolerant shrimp as compared to the ammonia-sensitive shrimp across all four families (Fig. 6).

\section{Discussion}

This study was aimed at investigating the ammonia tolerance in L.vannamei by QTL analysis. A high-density genetic map of $L$. vannamei was constructed using SLAF-seq, and a QTL associated with ammonia tolerance was identified as well as a putative candidate gene associated with ammonia tolerance.

The genome of L. vannamei is large $(\sim 2.45 \mathrm{~Gb})$ [32]. Whole-genome deep resequencing is relatively costly for large genomes and is often not necessary for gene/QTL mapping $[43,44]$. In recent years, several genetic linkage maps based on SNPs were constructed and QTL analyses were conducted in $L$. vannamei [30, 45-47]. Yu et al. constructed a high-density genetic map for L. vannamei and detected several QTLs for body weight and body length [30]. Yang et al. mapped the sex determination region in L. vannamei based on the data used for high-density linkage map construction [45]. Du et al.mapped a QTL for $L$. vannamei gender using a gene-based SNP linkage map [46]. In this study, a highdensity genetic map of $L$. vannamei was constructed using SLAF-seq, which is an effective method for discovering large numbers of SNPs and to perform large-scale genotyping [20]. Compared to traditional methods of genetic map construction (e.g., random amplified polymorphic DNA (RAPD), amplified fragment length polymorphism (AFLP), and simple sequence repeat (SSR)), the SLAF-seq method has several advantages for largescale SNP discovery and genotyping: high density, high throughput, high efficiency, and low cost [48]. Previous studies have developed genetic maps of $L$. vannamei using RAPD, AFLP, and SSR, but in these maps, the average distance between adjacent markers was $1-5 \mathrm{cM}$ [49-52]. The average distance between adjacent markers in the SLAF-seq genetic maps of $L$. vannamei in this study was substantially shorter $(0.34 \mathrm{cM})$. Notably, the average distance between adjacent markers found here was also less than in a previously reported SLAF-seq genetic map of L. vannamei $(0.75 \mathrm{cM})$ [30], possibly because a larger sample size and a greater sequencing depth were used. However, the number of LGs in the genetic map of $L$. vannamei in this study (44) was consistent with the number of LGs in the previously 


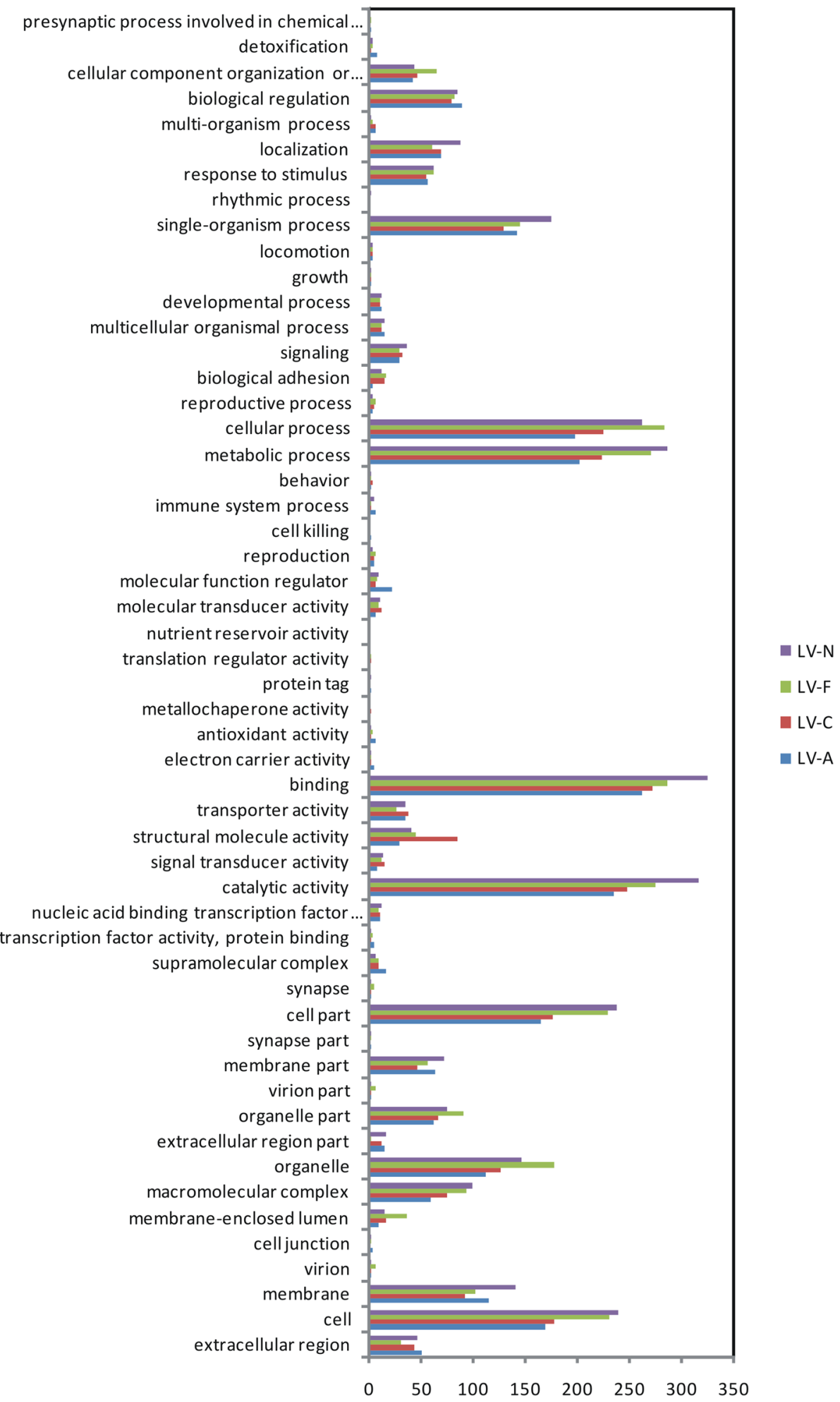

Fig. 5 Gene Ontology (GO) classifications of the putative functions of the differentially expressed genes between the most ammonia-tolerant and the most ammonia-sensitive individuals across four families of Litopenaeus vannamei (LV-A, LV-C, LV-F, and LV-N) 


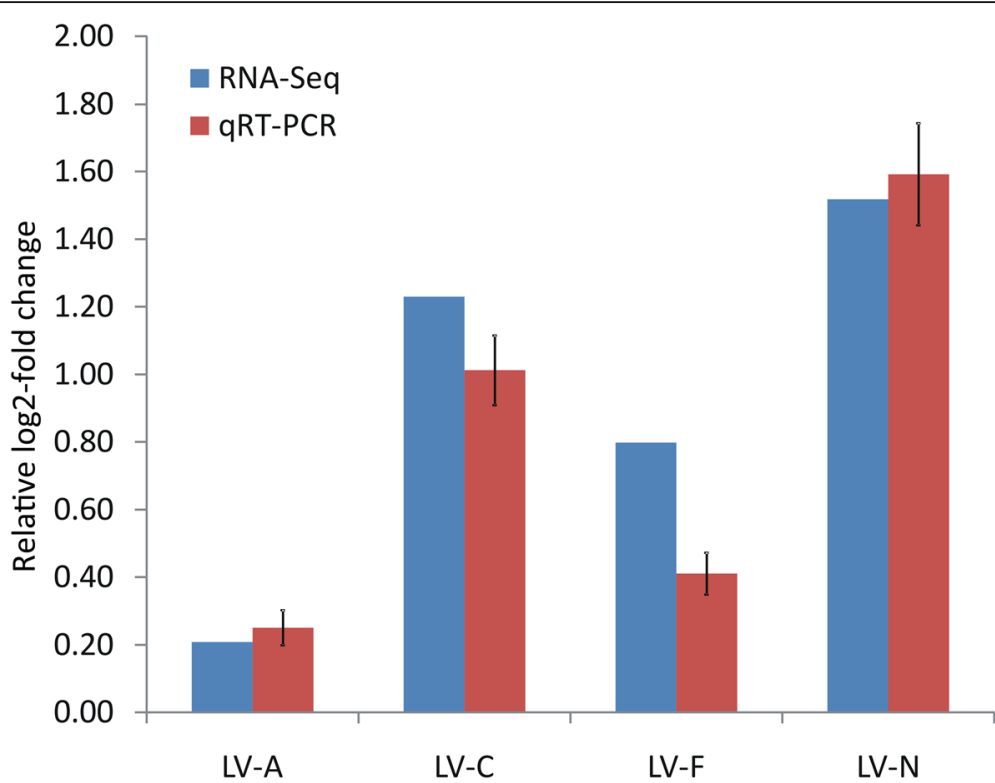

Fig. 6 Expression of LOC113809108 gene from the transcriptomic analysis validated by qRT-PCR. Expression of LOC113809108 gene was detected in the most ammonia-tolerant and the most ammonia-sensitive individuals from four families of Litopenaeus vannamei (LV-A, LV-C, LV-F, and LV$\mathrm{N})$. Data were normalized to $18 \mathrm{~s}$ rRNA as the reference and presented as a relative log2-fold change to validate the transcriptomic analysis results. Error bars show the standard deviation of three technical replicates

reported genetic map of L. vannamei [30]. This indicated that $L$. vannamei had 44 chromosomes, which was first reported by CamposRamos [53]. As far as we know, this is the highest density genetic linkage map L. vanna$m e i$, and it is very useful for comparative analysis of genomic synteny, QTL mapping, positioning of candidate genes, and marker-assisted selection.

As most animals cannot self-fertilize, it is difficult to develop common populations for genetic mapping (e.g., F2, recombinant inbred line (RIL), and nearlyisogenic line (NIL) populations). Therefore, an F1 population of L. vannamei was used to construct the genetic map, relying on a pseudo-testcross strategy. This strategy was based on the selection of single-dose markers present in one parent and absent in the other, and carried at a 1:1 ratio by the F1 offspring [54]. Therefore, gamete separation in each individual can be directly analyzed. The pseudo-testcross strategy has been widely used to construct animal F1 populations for genetic mapping [55-
58]. In this study, an F1 population was developed using one ammonia-tolerant male parent (the result of 10 generations of selective breeding) and one female shrimp from a common family.

Previous studies have suggested that the size of the mapped population might affect the accuracy of the genetic map and the QTL analysis, and have shown that genetic map accuracy increases with the size of the population used [59]. Specifically, populations of $>200$ individuals are considered sufficient for the construction of accurate genetic maps [59]. Thus, 284 randomlyselected individuals from the F1 population were used to construct the linkage map. However, the determination of shrimp survival time during the ammonia stress experiment depended on human observation, and thus may not have been perfectly accurate. To reduce the possible impacts of measurement inaccuracies on the QTL analysis, a relatively large F1 population was used. This larger population increased the accuracy of the

Table 2 RNA-Seq analysis showing expression of gene LOC113809108 in the most ammonia-tolerant and the most ammoniasensitive individuals across four families of Litopenaeus vannamei (LV-A, LV-C, LV-F, and LV-N)

\begin{tabular}{|c|c|c|c|c|c|c|c|c|}
\hline \multirow[t]{2}{*}{ Family } & \multicolumn{8}{|c|}{ Gene LOC113809108 expression level } \\
\hline & $\begin{array}{l}\text { Sensitive group } \\
\text { (FPKM) }\end{array}$ & $\begin{array}{l}\text { Sensitive group } \\
\text { (count) }\end{array}$ & $\begin{array}{l}\text { Tolerant group } \\
\text { (FPKM) }\end{array}$ & $\begin{array}{l}\text { Tolerant group } \\
\text { (count) }\end{array}$ & FDR & $\begin{array}{l}\text { Log2-fold } \\
\text { Change }\end{array}$ & Regulated & Difference \\
\hline LV-A & 112.8410 & 3239 & 130.2663 & 2561 & 0.9589 & 0.2072 & up & Normal \\
\hline LV-C & 63.2980 & 1651 & 148.3678 & 2444 & 0.0002 & 1.2289 & up & Significant \\
\hline LV-F & 70.4520 & 1378 & 122.4527 & 2258 & 0.9402 & 0.7975 & up & Normal \\
\hline LV-N & 114.9725 & 2659 & 329.0251 & 8025 & 0.0001 & 1.5169 & up & Significant \\
\hline
\end{tabular}


QTL mapping, compensating for any instances of human error in survival time measurement.

Ammonia stress is one of the biggest challenges facing shrimp aquaculture. Ammonia not only has a direct lethal effect on shrimp [7], but also inhibits the shrimp immune system and increases sensitivity to pathogens [60]. Breeding new varieties of ammonia-tolerant shrimp is therefore an important target of the shrimp aquaculture industry. Some researches on shrimp ammonia tolerance have been carried out in recent years. Lu et al. found 12 SNPs associated with ammonia tolerance in $L$. vannamei using marker-trait correlation analyses, and these SNPs were identified in the thrombospondin gene and X-box binding protein 1 gene [15]. At the same time, Lu et al. identified 202 differentially expressed proteins (DEPs) between ammonia-tolerant and ammoniasensitive $L$. vannamei using a comparative proteome analysis based on iTRAQ technique, and $77.8 \%$ of the DEPs were reported mainly involving in immune defense and stress tolerant in crustacean species [16]. Here, a QTL for ammonia-tolerance, located on LG19 at $169.09-169.49 \mathrm{cM}$ was identified, that explained 7.41$8.46 \%$ of the phenotypic variation in ammonia tolerance. To the best of our knowledge, this is the first QTL for ammonia-tolerance reported in shrimp. However, having one QTL that explains about $8 \%$ of the variance indeed is not enough to initiate genetic breeding. Therefore in future research, the cutoff of LOD score can be relaxed to get more QTLs, intervals, and candidate DEGs. And then, more candidate genes related to ammonia tolerance should be identified. Moreover, independent families should be used to verify whether the QTL is common across different populations.

A QTL usually spans a large chromosomal region and may contain hundreds of genes. Therefore, in order to identify functional genes associated with ammonia tolerance, the transcriptomes of $4 \mathrm{~L}$. vannamei families (LV$\mathrm{N}, \mathrm{LV}-\mathrm{A}, \mathrm{LV}-\mathrm{C}$, and LV-F) with different genetic backgrounds were sequenced. Combining QTL mapping and gene expression analysis, we identified a single DEG (LOC113809108), located in the QTL interval, that was annotated as an ATP synthase $g$ subunit and was significantly upregulated in the ammonia-tolerant LV-N and LV-C shrimp. The ATP synthase g subunit is located in F0 portion of ATP synthase, which consists of a membrane-integrated portion (F0 complex) and a membrane-protruding portion (F1 complex) [61]. The ATP synthase $g$ subunit is essential for the formation of the F0 complex [61]. In the previous studies $[15,16]$, ATP synthase has not been identified as being related to shrimp ammonia tolerance. ATP synthase is a doublemotor enzyme that is involved in ATP synthesis, ATP hydrolysis-dependent processes, and the regulation of the proton gradient across some membrane-dependent systems [62]. Several studies have shown that ammonia excretion in aquatic animals is associated with $\mathrm{Na}^{+} / \mathrm{K}^{+}$ATPase, which is mainly located on the basolateral membrane of branchial cells; $\mathrm{NH}_{4}{ }^{+}$is excreted into the environment when $\mathrm{K}^{+}$is replaced by $\mathrm{NH}_{4}{ }^{+}$via the $\mathrm{Na}^{+}$/ $\mathrm{NH}_{4}{ }^{+}$exchanger [63-65]. Indeed, a previous study suggested that in L. vannamei, high ammonia tolerance was mainly a result of improved ammonia excretion and detoxification, as well as an accelerated energy metabolism [17]. Therefore, we speculate that ATP synthesis might affect the ammonia tolerance of $L$. vannamei by regulating ATP synthesis and controlling cellular ammonia excretion. The results would provide useful information for further study of the molecular mechanisms of ammonia adaptive strategies in shrimps.

\section{Conclusions}

In this study, we constructed a high-density genetic map of L. vannamei and identified a QTL for ammonia tolerance. By combining QTL and transcriptome analyses, we identified a candidate gene associated with ammonia tolerance. The results help us better understand the molecular mechanism of ammonia tolerance in shrimp. Our work provides the basis for future genetic studies focused on molecular marker-assisted selective breeding.

\section{Supplementary Information}

The online version contains supplementary material available at https://doi. org/10.1186/s12864-020-07254-X.

\section{Additional file 1: Table S1. Median lethal concentration of $\mathrm{NH} 4 \mathrm{Cl}$ for Litopenaeus vannamei families LV-A, LV-C, LV-F, and LV-N. \\ Additional file 2: Table S2. Summary of the SLAF sequencing of Oryza sativa japonica.}

Additional file 3: Table S3. Basic information of the male map.

Additional file 4: Table S4. Basic information of the female map.

Additional file 5: Table S5. Basic information of the sex-average map

Additional file 6: Table S6. Statistics of the number of differentially expressed genes (DEGs) between the most ammonia-sensitive and most ammonia-tolerant shrimp in the four experimental families.

Additional file 7: Table S7. DEGs within the QTL interval and their expression levels and annotations

Additional file 8: Figure $\mathbf{S 1}$. The accumulated mortality rate of shrimp in LV-N family under an acute ammonia stress (ammonia- $\mathrm{N}$ concentration of $345.94 \mathrm{mg} / \mathrm{L}$, temperature of $27.0 \pm 0.5^{\circ} \mathrm{C}$, pH of $8.1 \pm 0.2$, salinity of $30.2 \%$, and dissolved oxygen of $6-8 \mathrm{mg} / \mathrm{L}$ ).

\footnotetext{
Abbreviations

CM: Centimorgans; SLAF-seq: Specific length amplified fragment sequencing; QTL: Quantitative trait locus; ammonia-N: Ammonia nitrogen; SNPs: Single nucleotide polymorphisms; PCRs: Polymerase chain reactions; LOD: Logarithm of odds; qRT-PCR: Quantitative real-time PCR; FPKM: Fragments per kilobase of transcript per million mapped reads; FDR: False discovery rate; DEGs: Differentially expressed genes; Gb: Gigabases; Mb: Megabases; LGs: Linkage groups
}

\section{Acknowledgments}

We thank the Guangxi Shrimp Breeding Engineering Technology Research Center for providing animal materials. 


\section{Authors' contributions}

YZ and QL conceived the study. DZ and CY wrote the manuscript and contributed in the bioinformatics analysis. QL, WZ, XC, and MP conducted the experiments and contributed for raw data analysis. XC, YL, HW, HL, and $J L$ contributed in the bioinformatics analysis. All authors read and approved the final manuscript.

\section{Funding}

This work was supported by the scientific and technological innovation major project of guangxi (aa17204080-1), scientific and technological innovation major base of guangxi (aa17204088-1), guangxi scientific research and technology development project (ab16380189), the national modern agriculture industry technology system project (nycytxgxcxtd-14-01), and the national modern agroindustry technology research system (cars-48).

\section{Availability of data and materials}

All data generated or analysed during this study are included in this published article and its supplementary information files. Raw SLAF sequencing reads are deposited in NCBI database under the accession numbers PRJNA545592. Raw RNA-seq are deposited in NCBI database under the accession numbers SRR9822091, SRR9822090, SRR9822095, SRR9822094, SRR9822098, SRR9822099, SRR9822093, and SRR9822085.

\section{Ethics approval and consent to participate}

Not applicable.

\section{Consent for publication}

Not applicable.

\section{Competing interests}

The authors declare they have no competing interests.

\section{Author details}

'Guangxi Key Laboratory of Aquatic Genetic Breeding and Healthy Aquaculture, Guangxi Academy of Fishery Sciences, Nanning 530021, China. ${ }^{2}$ Key Lab of Freshwater Animal Breeding, Key Laboratory of Agricultural Animal Genetics, Breeding and Reproduction, Ministry of Education, College of Fishery, Huazhong Agriculture University, Wuhan 430070, China. ${ }^{3}$ Life Science Research Institute, Guangxi University, Nanning 530004, China.

\section{Received: 3 December 2019 Accepted: 18 November 2020}

\section{Published online: 02 December 2020}

\section{References}

1. Cruz P, Ibarra AM, Mejia-Ruiz H, Gaffney PM, Perez-Enriquez R. Genetic variability assessed by microsatellites in a breeding program of Pacific white shrimp (Litopenaeus vannamei). Mar Biotechnol (NY). 2004;6(2):157-64.

2. Javahery S, Noori A, Hoseinifar SH. Growth performance, immune response, and digestive enzyme activity in Pacific white shrimp, Penaeus vannamei Boone, 1931, fed dietary microbial lysozyme. Fish Shellfish Immunol. 2019; 92:528-35.

3. Alagappan KM, Deivasigamani B, Somasundaram ST, Kumaran S. Occurrence of Vibrio parahaemolyticus and its specific phages from shrimp ponds in east coast of India. Curr Microbiol. 2010;61(4):235-40.

4. Sun CB, Wang G, Chan SF. Effects of artificial infection of Litopenaeus vannamei by Micrococcus lysodeikticus and WSSV on the activity of immunity related enzymes. Fish Shellfish Immunol. 2015;46(2):778-86.

5. Marazza D, Bornens $P$, Le Gal Y. Effect of ammonia on survival and adenylate energy charge in the shrimp Palaemonetes varians. Ecotoxicol Environ Saf. 1996;34(2):103-8.

6. Frias-Espericueta MG, Harfush-Melendez M, Paez-Osuna F. Effects of ammonia on mortality and feeding of postlarvae shrimp Litopenaeus vannamei. Bull Environ Contam Toxicol. 2000;65(1):98-103.

7. Qiu L, Shi X, Yu S, Han Q, Diao X, Zhou H. Changes of Ammoniametabolizing enzyme activity and gene expression of two strains in shrimp Litopenaeus vannamei under Ammonia stress. Front Physiol. 2018:9:211.

8. Liang Z, Liu R, Zhao D, Wang L, Sun M, Wang M, Song L. Ammonia exposure induces oxidative stress, endoplasmic reticulum stress and apoptosis in hepatopancreas of pacific white shrimp (Litopenaeus vannamei). Fish Shellfish Immunol. 2016;54:523-8.
9. Lu X, Kong J, Luan S, Dai P, Meng X, Cao B, Luo K. Transcriptome analysis of the Hepatopancreas in the Pacific white shrimp (Litopenaeus vannamei) under acute Ammonia stress. PLoS One. 2016;11(10):e0164396.

10. Lu X, Luan S, Cao B, Meng X, Sui J, Dai P, Luo K, Shi X, Hao D, Han G, et al. Estimation of genetic parameters and genotype-by-environment interactions related to acute ammonia stress in Pacific white shrimp (Litopenaeus vannamei) juveniles at two different salinity levels. PLoS One. 2017;12(3):e0173835

11. Duan $Y$, Zhang $Y$, Dong $H$, Wang $Y$, Zheng $X$, Zhang J. Effect of dietary Clostridium butyricum on growth, intestine health status and resistance to ammonia stress in Pacific white shrimp Litopenaeus vannamei. Fish Shellfish Immunol. 2017;65:25-33.

12. Valencia-Castaneda G, Frias-Espericueta MG, Vanegas-Perez RC, PerezRamirez JA, Chavez-Sanchez MC, Paez-Osuna F. Acute toxicity of Ammonia, nitrite and nitrate to shrimp Litopenaeus vannamei Postlarvae in low-salinity water. Bull Environ Contam Toxicol. 2018;101(2):229-34.

13. Campbell NR, LaPatra SE, Overturf K, Towner R, Narum SR. Association mapping of disease resistance traits in rainbow trout using restriction site associated DNA sequencing. G3 (Bethesda). 2014;4(12):2473-81.

14. Yu Y, Liu J, Li F, Zhang X, Zhang C, Xiang J. Gene set based association analyses for the WSSV resistance of Pacific white shrimp Litopenaeus vannamei. Sci Rep. 2017;7:40549.

15. Lu X, Kong J, Meng X, Cao B, Luo K, Dai P, Luan S. Identification of SNP markers associated with tolerance to ammonia toxicity by selective genotyping from de novo assembled transcriptome in Litopenaeus vannamei. Fish Shellfish Immunol. 2018;73:158-66.

16. Lu X, Luan S, Dai P, Meng X, Cao B, Luo K, Kong J. iTRAQ-based comparative proteome analysis for molecular mechanism of defense against acute ammonia toxicity in Pacific white shrimp Litopenaeus vannamei. Fish Shellfish Immunol. 2018;74:52-61.

17. Xiao J, Li QY, Tu JP, Chen XL, Chen XH, Liu QY, Liu H, Zhou XY, Zhao YZ, Wang $\mathrm{HL}$. Stress response and tolerance mechanisms of ammonia exposure based on transcriptomics and metabolomics in Litopenaeus vannamei. Ecotoxicol Environ Saf. 2019:180:491-500.

18. Lu X, Luan S, Dai P, Luo K, Chen B, Cao B, Sun L, Yan Y, Kong J. Insights into the molecular basis of immunosuppression and increasing pathogen infection severity of ammonia toxicity by transcriptome analysis in pacific white shrimp Litopenaeus vannamei. Fish Shellfish Immunol. 2019;88:528-39.

19. Zhu M, Zhao S. Candidate gene identification approach: progress and challenges. Int J Biol Sci. 2007;3(7):420-7.

20. Sun X, Liu D, Zhang X, Li W, Liu H, Hong W, Jiang C, Guan N, Ma C, Zeng H, et al. SLAF-seq: an efficient method of large-scale de novo SNP discovery and genotyping using high-throughput sequencing. PLoS One. 2013;8(3): e58700.

21. Qian W, Fan G, Liu D, Zhang H, Wang X, Wu J, Xu Z. Construction of a highdensity genetic map and the $X / Y$ sex-determining gene mapping in spinach based on large-scale markers developed by specific-locus amplified fragment sequencing (SLAF-seq). BMC Genomics. 2017;18(1):276.

22. Zhang $Y$, Wang $L$, Xin H, Li D, Ma C, Ding $X$, Hong W, Zhang $X$. Construction of a high-density genetic map for sesame based on large scale marker development by specific length amplified fragment (SLAF) sequencing. BMC Plant Biol. 2013;13:141.

23. Zhu Y, Yin Y, Yang K, Li J, Sang Y, Huang L, Fan S. Construction of a highdensity genetic map using specific length amplified fragment markers and identification of a quantitative trait locus for anthracnose resistance in walnut (Juglans regia L.). BMC Genomics. 2015;16:614

24. Li B, Tian L, Zhang J, Huang L, Han F, Yan S, Wang L, Zheng H, Sun J. Construction of a high-density genetic map based on large-scale markers developed by specific length amplified fragment sequencing (SLAF-seq) and its application to QTL analysis for isoflavone content in Glycine max. BMC Genomics. 2014;15:1086.

25. Wei Q, Wang Y, Qin X, Zhang Y, Zhang Z, Wang J, Li J, Lou Q, Chen J. An SNP-based saturated genetic map and QTL analysis of fruit-related traits in cucumber using specific-length amplified fragment (SLAF) sequencing. BMC Genomics. 2014;15:1158.

26. Jiang B, Liu W, Xie D, Peng Q, He X, Lin Y, Liang Z. High-density genetic map construction and gene mapping of pericarp color in wax gourd using specific-locus amplified fragment (SLAF) sequencing. BMC Genomics. 2015; 16:1035.

27. Zhao Z, Gu H, Sheng X, Yu H, Wang J, Huang L, Wang D. Genome-wide single-nucleotide polymorphisms discovery and high-density genetic map 
construction in cauliflower using specific-locus amplified fragment sequencing. Front Plant Sci. 2016;7:334.

28. Tao A, Huang L, Wu G, Afshar RK, Qi J, Xu J, Fang P, Lin L, Zhang L, Lin P. High-density genetic map construction and QTLs identification for plant height in white jute (Corchorus capsularis L.) using specific locus amplified fragment (SLAF) sequencing. BMC Genomics. 2017;18(1):355.

29. Liu C, Zhou Q, Dong L, Wang H, Liu F, Weng J, Li X, Xie C. Genetic architecture of the maize kernel row number revealed by combining QTL mapping using a high-density genetic map and bulked segregant RNA sequencing. BMC Genomics. 2016;17(1):915.

30. Yu Y, Zhang X, Yuan J, Li F, Chen X, Zhao Y, Huang L, Zheng H, Xiang J. Genome survey and high-density genetic map construction provide genomic and genetic resources for the Pacific white shrimp Litopenaeus vannamei. Sci Rep. 2015;5:15612.

31. Gilcreas FW. Future of standard methods for the examination of water and wastewater. Health Lab Sci. 1967;4(3):137-41.

32. Zhang X, Yuan J, Sun Y, Li S, Gao Y, Yu Y, Liu C, Wang Q, Lv X, Ma KY, et al. Penaeid shrimp genome provides insights into benthic adaptation and frequent molting. Nat Commun. 2019;10(1):356.

33. Li H, Durbin R. Fast and accurate short read alignment with burrowswheeler transform. Bioinformatics. 2009;25(14):1754-60.

34. Liu D, Ma C, Hong W, Huang L, Liu M, Liu H, Zeng H, Deng D, Xin H, Song $\mathrm{J}$, et al. Construction and analysis of high-density linkage map using highthroughput sequencing data. PLoS One. 2014;9(6):e98855.

35. Broman KW, Gatti DM, Simecek P, Furlotte NA, Prins P, Sen S, Yandell BS, Churchill GA. R/qt2: software for mapping quantitative trait loci with highdimensional data and multiparent populations. Genetics. 2019;211(2):495502.

36. Gardiner SE, Norelli UL, de Silva N, Fazio G, Peil A, Malnoy M, Horner M, Bowatte $D$, Carlisle C, Wiedow $C$, et al. Putative resistance gene markers associated with quantitative trait loci for fire blight resistance in Malus 'Robusta 5' accessions. BMC Genet. 2012;13:25.

37. Kim D, Langmead B, Salzberg SL. HISAT: a fast spliced aligner with low memory requirements. Nat Methods. 2015;12(4):357-60.

38. Mortazavi A, Williams BA, McCue K, Schaeffer L, Wold B. Mapping and quantifying mammalian transcriptomes by RNA-Seq. Nat Methods. 2008;5(7): $621-8$.

39. Robinson MD, McCarthy DJ. Smyth GK: edgeR: a bioconductor package for differential expression analysis of digital gene expression data. Bioinformatics. 2010;26(1):139-40.

40. Singh VK, Mangalam AK, Dwivedi S, Naik S. Primer premier: program for design of degenerate primers from a protein sequence. BioTechniques. 1998;24(2):318-9.

41. Zhang Q, Li F, Zhang X, Dong B, Zhang J, Xie Y, Xiang J. cDNA cloning, characterization and expression analysis of the antioxidant enzyme gene, catalase, of Chinese shrimp Fenneropenaeus chinensis. Fish Shellfish Immunol. 2008;24(5):584-91.

42. Livak KJ, Schmittgen TD. Analysis of relative gene expression data using real-time quantitative $P C R$ and the 2(-Delta Delta $C(T))$ method. Methods. 2001;25(4):402-8.

43. Xu X, Chao J, Cheng X, Wang R, Sun B, Wang H, Luo S, Wu T, Li Y. Mapping of a novel race specific resistance gene to Phytophthora root rot of pepper (Capsicum annuum) using bulked Segregant analysis combined with specific length amplified fragment sequencing strategy. PLoS One. 2016; 11(3):e0151401.

44. Wang G, Chen B, Du H, Zhang F, Zhang H, Wang Y, He H, Geng S, Zhang X. Genetic mapping of anthocyanin accumulation-related genes in pepper fruits using a combination of SLAF-seq and BSA. PLoS One. 2018;13(9): e0204690.

45. Yu Y, Zhang X, Yuan J, Wang Q, Li S, Huang H, Li F, Xiang J. Identification of sex-determining loci in Pacific white shrimp Litopeneaus vannamei using linkage and association analysis. Mar Biotechnol. 2017;19:277-86.

46. Du ZQ, Ciobanu DC, Onteru SK, Gorbach D, Mileham AJ, Jaramillo G, Rothschild MF. A gene-based SNP linkage map for pacific white shrimp, Litopenaeus vannamei. Anim Genet. 2010;41(3):286-94.

47. Du Z, Onteru S, Gorbach D, Rothschild M. A SNP genetic map for Pacific white shrimp (Litopenaeus vannamei). Anim Industry Rep. 2010;656(1):35.

48. Liang D, Chen M, Qi X, Xu Q, Zhou F, Chen X. QTL mapping by SLAF-seq and expression analysis of candidate genes for aphid resistance in cucumber. Front Plant Sci. 2016:7:1000.
49. Garcia DK, Dhar AK, Alcivar-Warren A. Molecular analysis of a RAPD marker (B20) reveals two microsatellites and differential mRNA expression in Penaeus vannamei. Mol Mar Biol Biotechnol. 1996;5(1):71-83.

50. Perez F, Ortiz J, Zhinaula M, Gonzabay C, Calderon J, Volckaert FA. Development of EST-SSR markers by data mining in three species of shrimp: Litopenaeus vannamei, Litopenaeus stylirostris, and Trachypenaeus birdy. Mar Biotechnol (NY). 2005;7(5):554-69.

51. Andriantahina F, Liu X, Huang H. Genetic map construction and quantitative trait locus (QTL) detection of growth-related traits in Litopenaeus vannamei for selective breeding applications. PLoS One. 2013;8(9):e75206.

52. Zhang L, Yang C, Zhang Y, Li L, Zhang X, Zhang Q, Xiang J. A genetic linkage map of Pacific white shrimp (Litopenaeus vannamei): sex-linked microsatellite markers and high recombination rates. Genetica. 2007;131(1): 37-49.

53. CamposRamos R. Chromosome studies on the marine shrimps Penaeus vannamei and P-californiensis (Decapoda). J Crustac Biol. 1997;17(4):666-73.

54. Grattapaglia D, Sederoff R. Genetic linkage maps of Eucalyptus grandis and Eucalyptus urophylla using a pseudo-testcross: mapping strategy and RAPD markers. Genetics. 1994;137(4):1121-37.

55. Grattapaglia D, Bertolucci FL, Sederoff RR. Genetic mapping of QTLs controlling vegetative propagation in Eucalyptus grandis and E. urophylla using a pseudo-testcross strategy and RAPD markers. TAG Theor Appl Genet Theor Angewandte Gen. 1995;90(7-8):933-47.

56. Porceddu A, Albertini E, Barcaccia G, Falistocco E, Falcinelli M. Linkage mapping in apomictic and sexual Kentucky bluegrass (Poa pratensis L.) genotypes using a two way pseudo-testcross strategy based on AFLP and SAMPL markers. TAG Theor Appl Genet Theor Angewandte Gen. 2002; 104(2-3):273-80.

57. Liao M, Zhang L, Yang G, Zhu M, Wang D, Wei Q, Zou G, Chen D. Development of silver carp (Hypophthalmichthys molitrix) and bighead carp (Aristichthys nobilis) genetic maps using microsatellite and AFLP markers and a pseudo-testcross strategy. Anim Genet. 2007;38(4):364-70.

58. Xie W, Zhang X, Cai H, Huang L, Peng Y, Ma X. Genetic maps of SSR and SRAP markers in diploid orchardgrass (Dactylis glomerata L.) using the pseudo-testcross strategy. Genome. 2011;54(3):212-21.

59. MFdS AFI, Silva I L d C e, Cosme Damião Cruz I. Estimating the effects of population size and type on the accuracy of genetic maps. Genet Mol Biol. 2006;29(1):187-92

60. Jiang L, Feng J, Ying R, Yin F, Pei S, Lu J, Cao Y, Guo J, Li Z. Individual and combined effects of ammonia-N and sulfide on the immune function and intestinal microbiota of Pacific white shrimp Litopenaeus vannamei. Fish Shellfish Immunol. 2019;92:230-40.

61. Fujikawa M, Sugawara K, Tanabe T, Yoshida M. Assembly of human mitochondrial ATP synthase through two separate intermediates, F1-c-ring and b-e-g complex. FEBS Letter. 2015;589(19 Pt B):2707-12.

62. Hong S, Pedersen PL: ATP synthase and the actions of inhibitors utilized to study its roles in human health, disease, and other scientific areas. Microbiol Mol Biol Rev. 2008: 72(4):590-641, Table of Contents.

63. Wilkie MP. Mechanisms of ammonia excretion across fish gills. Comp Biochem Physiol Part A. 1997;118A(1):39-50.

64. Lucu C, Flik G. Na+-K+-ATPase and Na+/Ca2+ exchange activities in gills of hyperregulating Carcinus maenas. Am J Phys. 1999;276(2):R490-9.

65. Weihrauch D, Morris S, Towle DW. Ammonia excretion in aquatic and terrestrial crabs. J Exp Biol. 2004;207(Pt 26):4491-504.

\section{Publisher's Note}

Springer Nature remains neutral with regard to jurisdictional claims in published maps and institutional affiliations. 